\title{
XXI. On the probability of ionization and radiation of gas molecules due to collision with electrons
}

\section{K.F. Nesturch}

To cite this article: K.F. Nesturch (1915) XXI. On the probability of ionization and radiation of gas molecules due to collision with electrons , Philosophical Magazine Series 6, 30:176, 244-248, DOI: $10.1080 / 14786440808635391$

To link to this article: http://dx.doi.org/10.1080/14786440808635391

曲 Published online: 08 Apr 2009.

Submit your article to this journal \lceil

Џ Article views: 5

Q View related articles $\sqsubset$ 
XXI. On the Probability of Ionization and Radiation of Gas Molecules due to Collision with Electrons. By K. F. Nesturch *.

A $\mathrm{S}$ recently shown by Franck and Hertz $\dagger$, the electrons $A$ in electropositive gases ( $\mathrm{He}, \mathrm{Ne}, \mathrm{Ar}$, also mercury vapour), set in motion by the action of an electric field, collide elastically with neutral gas molecules. Consequently when passing a fall of potential, the electrons accumulate kinetic energy independently of the number of collisions on their way, $i$. e. independently of the pressure of the gas.

When an electron has obtained a certain amount of energy, its next collision with a gas molecule is an inelastic, one; it loses its energy, which is then transferred to the molecule. The amount of energy, possessed by the electron at the moment of the inelastic collision, is a characteristic quantity of the gas, the corresponding fall of potential being the "ionizing potential" of the latter.

Franck and Hertz have brought forward numerous arguments $\ddagger$ in favour of the view that the energy, which an electron loses by this inelastic shock, can either ionize the molecule or force it to emit radiation of a definite wavelength, but that both processes cannot occur simultaneously.

To this it may be added that according to the model of the atom, recently designed by Dr. Bohr $\$$, the possibility of such transformation of energy is very obvious in both cases.

It is possible to suppose then that the ratio of probabilities of ionization and radiation is a characteristic property of the gas. In this article the author will attempt to indicate a method for a rough determination of the numerical values of the above probabilities for electropositive gases only.

In the first place a formula is to be given for the value of the electric current between the two plane electrodes in a homogeneous field, the gradient of potential being $\mathrm{X}$. The two following assumptions are made for the sake of simplicity. 1. The "ionizing distance," which the electron must pass before it has gained the amount of energy sufficient to experience an inelastic shock, must be great as compared with the mean free path of the electrons. 2. $l$, the distance between the plates, must be an exact multiple of the ionizing

* Communicated by the Author, having been read before the Russian Physico-Chemical Society at the meeting of 22 December, 1914.

f Verh.d. D. Phys. Ges. xv. (1913) : xvi. p. 45 i (1914).

$\ddagger$ L. c. and Verh. d. D. Phys. Ges. xvi. p. 612 (1914).

\$ Phil. Mag. xxvi, p. 1 (1913). 
distance; of course the same relation must exist between the potential difference $\mathrm{Xl}$, on the electrodes, and the ionizing potential $\mathrm{V}$.

Let $\gamma$ be the probability of the ionization of the gas molecule due to an inelastic collision. Then the probability of the emission of light will be equal to $1-\gamma$.

Suppose that $n_{0}$ electrons set free by some external cause (for instance, by photoelectric effect) are made to travel from the cathode towards the anode plate. After having passed the fall of potential $\mathrm{V}$ they will inelastically collide with the gas molecules and produce $n_{0} \gamma$ new pairs of ions. Therefore in the electric field there will be $n_{0}(1+\gamma)$ electrons, and after a new series of collisions the number will be increased to $n_{0}(1+\gamma)^{2}$, and so on.

It is obvious that the total number of electrons reaching the positive plate will be equal to

$$
n_{0}(1+\gamma)^{\frac{\mathrm{x}}{\mathrm{V}} l} \text {. . . . . . . }
$$

In Prof. Townsend's theory* the same quantity is denoted by the expression

$$
n_{0} e^{a l}, \text {. . . . . . . . }
$$

where $\alpha$ is the number of ions which an electron produces by collision in going through a centimetre of the gas. The numerical values of a were experimentally determined over a wide range of gradient $X$ and pressure $p$ by Prof. Townsend and his pupils. These values can be easily obtained from the curves, representing $\frac{\alpha}{p}$ as a function of $\frac{\mathrm{X}}{p}$.

By means of the expressions (1) and (2) we can write an equation for the determination of $\gamma$,

$$
\log \operatorname{nat}(1+\gamma)=\frac{\mathrm{V}}{\mathrm{X}} a . . \quad . \quad . \quad .
$$

This equation may be considered in a slightly modified form

$$
\alpha=\frac{\mathrm{X}}{\mathrm{V}} \log \mathrm{nat}(1+\gamma), \ldots . . \quad \text {. . }
$$

which determines $\alpha$ as a linear function of $\mathrm{X}$. This formula holds good only when the above-mentioned limitation is made for the "ionizing distances." With increase of $X$ the "ionizing distances" are diminished and become comparable with the mean free paths of the electrons. In this case after

* J, S. Townsend, "The Theory of Ionization of Gases by Collision." 
an electron has gained an amount of energy, sufficiently great to be able to ionize, it must sometimes pass a considerable distance before it comes into collision with a gas molecule. We can make the probable assumption that, when such an electron overcharged with energy collides with a gas molecule, the electron newly formed by the process will have the initial velocity at the expense of the excess of energy of the parent electron. Therefore it will be further moved as if it were formed right on the plane, parallel to the cathode plate, where the parent electron had acquired the critical amount of energy sufficient for ionization. The movement of the latter, however, will be slower when compared with that assumed in the simplifer culculations of the expression (1).

Consequently for large values of $X$, the charge received by the anode and therefore the values of $\alpha$ are less than those calculated by means of the expression (1) and formula (4). As stated by Prof. Townsend*, the highest possible value of a does not depend upon $X$, and is equal to the number of free paths of the electron per centimetre of its way.

Therefore one might expect that the lines, representing $\alpha$ as a function of $X$ for large values of $X$, will become more and more concave towards the axis of abscissa. Further, since according to the formula (4) these lines ought to pass through the origin, they will have the form represented in fig. 1.

Fig. 1.

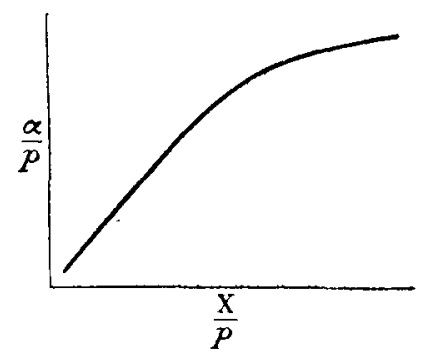

Fig. 2.

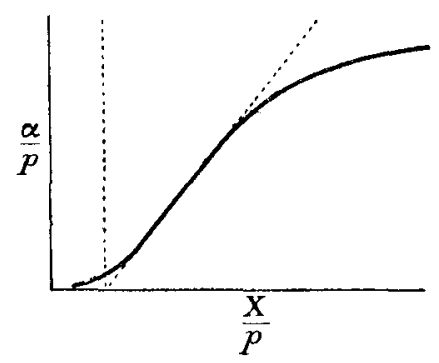

Experimental curves, however, have the shape given in fig. 2, and this shows that $\frac{\alpha}{p}$ is a linear function only for certain values of $\frac{\mathrm{X}}{p}$.

Such disagreement may bo due to different causes. Either 

the value of $\gamma$ for small $\frac{\mathrm{X}}{p}$ is not constant, or for small $\mathrm{X}$ an inelastic shock does not take place at every collision $p$

of the electron, possessing the critical amount of energy, with the gas molecule, but depends upon their relative position at the moment of the shock.

On the strength of the present experimental results it is impossible to explain the shape of the curve for small values of $\frac{\mathrm{X}}{p}$. Whatever cause determines the form of the curve, its infuence disappears with the increase of $\frac{\mathrm{X}}{p}$.

For the determination of values of $\gamma$ we obviously must take the straight part of the curve, prolong it as far as the intersection with the axis of abscissæ, and take this point for the new origin. In this way we can determine the value of $\alpha$ for a given $X$, and further, using for $V$ the data found by Franck and Hertz, we then can calculate the numerical value of $\gamma$ by means of the equation (3).

The described method has as yet been applied to argon and helium only, and the following results were obtained.

Argon. The point of intersection with the axis of abscissæ of the prolonged straight part of the curve corresponds to $\frac{\mathrm{X}}{\mathrm{p}}=30$. The ionizing potential of argon, according to Franck and Hertz, is equal to 12 volts. Thus $\gamma=$ about 0.4 .

Helium. In the case of helium the experimental results are not sufficiently definite. Gill and Pidduck in a careful investigation * have given a characteristic curve for the gas, containing 2 per cent. of impurities. The point of intersection for this curve is $\frac{X}{p}=8$. The ionizing potential of helium is 20.5 volts. The value of $\gamma$, calculated by means of these data, is about 0.9 . So far as pure helium is concerned, the results are not so certain, and the curve is not characteristic enough to permit the application of this method. There is no doubt, however, that the ionization increases with the purity of the gas, and the value $\gamma$ for the pure gas must consequently be greater than 0.9 .

From the difference of values of $\gamma$ for the two gases it is to be expected that under the impact of electrons helium will be more readily ionized than argon, while argon will be more capable of emitting light. The following observations, made by Collie and Ramsayt, may be considered as a confirmation

* Phil. Mag. xvi. p. 280 (1908).

† Proc. Roy. Soc. lix. pp. 257-270 (1896). 
of the above results. A small quantity of argon, present in helium when a discharge is sent through a Geissler tube, may be easily detected spectroscopically: but when some helium is present in argon its quantity must be considerably greater in order to give a distinct enough spectrum.

With regard to the electronegative gases, gases in which electrons lose their energy at every collision with the molecules, the analogous considerations are probably far inore complicated.

Pefrograd, Physical Institute of the University.

XXII. The Coefficient of End-Correction.-Part II. By P. J. Daniele, B.A., Assistant Professor in Applied Mathematics, The Rice Institute, Houston, Texas *.

$\$ 1$. TF an electrical current passes throngh a long cylin1 drical tube of conducting material, and then out into a large hemispherical volume of the same, the total resistance is proportional to the total length of the tube plus a certain multiple of the radius. This multiple is the coefficient of end-correction which we require to find.

Rayleigh in his 'Theory of Sound' found that

$$
\cdot 785<\text { this coefficient } k<\cdot 845 \text {. }
$$

In the previous paper with the same title the author assumed the normal current at the open end to be of a form

$$
A+B\left(1-\varpi^{2}\right)+C\left(1-\varpi^{2}\right)^{-1 / 3} \text {. }
$$

Thus it was found that

(1) if $\mathrm{B}$ is neglected, the approximate value of $k$ is 82171 ;

(2) considering $B$, the approximate value of $k$ is $\cdot 82168$. Then the method shows that the real value of $k<\cdot 82168$.

In this paper the author states a method by which an approximate value of $k$ can be found which is less than the real value. In fact

$$
-82141<\text { real value } k \text {. }
$$

Thus $k$ is confined between the narrow limits

$$
\cdot 82141<k<.82168 \text {. }
$$

The method by which Rayleigh found the lower limit $\cdot 785$ for $k$, was to assume a value for the potential $\mathrm{V}$ at the

\footnotetext{
* Commuvieated by the Author.
} 